\title{
The Influence Explore of Teresa Teng's Singing Style on Pop Music
}

\author{
Yan Li \\ Tianjin Conservatory of Music, Tianjin, China
}

Keywords: Teresa Teng, singing style, pop music, influence

\begin{abstract}
Teresa Teng, a talent singer star of China, who has sweet voice, had won high reputation not only in Hong Kong, Taiwan, Japan and Southeast Asia, but also made the people in mainland crazy about her music, and become a top-class person in Southeast Asia's popular music world. A large number of music works sang by Teresa Teng have become parts of classic Chinese songs, exerting a profound and meaningful effect. Based on the social background, this paper analyzed the music thought and the artistic characteristics of Teresa Teng's songs. Besides, the contribution and influence on pop music in China were summed up to help readers enjoy and understand Teresa Teng's songs.
\end{abstract}

\section{Introduction}

Teresa Teng is a legendary figure in the twentieth-century mainland Chinese pop music scene. She created a "Teresa Teng era" with singing voice, playing a decisive role in the development of pop music in Mainland China and becoming the most popular Taiwanese singer in the early days of reform and opening up. In retrospect of 30 years of reform and opening up, Teresa Teng music as a classic melody to become an important chapter to review the Golden Years. She is gentle and elegant, elegant and passionate song across national boundaries and nationalities is a mark of the times, but also beyond the continuation of the time. Until now, Teresa Teng and his classic songs are still classic pop music in our country, loved and respected by the people. Teresa was successful, she created a time belongs to her, but the popularity of any song or singer is not accidental, not to mention Teresa Teng is not just ephemeral Yao Yi Yan, but for a long time, a wide range of people occupy The phenomenon of popular music, communication technology is more developed today are rare, thought-provoking [1].

Teresa Teng's song represents an era. It should be said that the diversity of artistic ideas created the success of Teresa Teng, and Teresa's personal efforts also contributed to the development and progress of the arts. Although many singers still imitate Teresa Teng's acting style and singing, up to now, none of them can reproduce Teresa Teng's artistic accomplishments either because the artist's artistic accomplishments are not yet in place. More importantly, Teresa Teng's own unique art Temperament difficult to be imitated and beyond [1].

\section{Teresa Teng's artistic career}

The popularity of Teresa Teng's songs is related to many factors such as the specific historical conditions, the characteristics of the times and the social conditions of her life. However, the crucial point is that the style formed in her artistic career plays a decisive role.

\subsection{Local opera music enlightenment}

Teresa's interest in traditional Chinese opera raised her good musical feeling and demonstrated her talent and potential in the art field. She laid the foundation for her future professional performing arts. Taiwanese native Taiwanese opera and the Huangmei opera of mainland China are the images of Teresa Teng's childhood when most contact, the most affected folk art, especially the Huangmei opera. In 1964, at the "Huangmei Opera Song" competition organized by "CCTV", the 11-year-old Teresa Teng's antithesis film "Liang Shanbo and Zhu Yingtai" Teresa Teng blockbuster became the beginning of professional art road after her [2]. 


\subsection{The development of "swirling" style singing}

Zhou swirl is one of the pinnacles of early Chinese pop music, she created a generation of singing style with her own unique singing style. Teresa Teng is an admirer and follower of Zhou Xu. She not only covers many songs sung by Zhou Zhou, but also maintains and develops such conversational style of singing. Her voice is more mellow, euphemistic and singing skills also more mature and perfect. As their representative work, "Where the Regret Comes Again," Teresa inherited Zhou Xu's soft whisper, but Teresa Teng, who received professional vocal training, not only rounded Zhou's "sharpness" The expression of the song is also more soothing lyricism, this "more musical" "more artistic" interpretation of Teresa Teng can swiftly walk in the swirling singing style, although not the full reproduction of the old Shanghai luxury, but it is the combination of old and new , Inherit and innovate with the development of the times [2].

\subsection{Assimilate "Japan" and "American" style}

In 1973, Teresa Teng resolutely gave up the good momentum of development in Hong Kong and Taiwan and opened up only to Japan [1]. Teresa Teng not only mastered the genre of the ensemble song, but also into the elements of modern pop music to tune song-based "Airport" to make Teresa overnight fame, released in 1987 album "King of Enchantress" has been hailed as "Queen Endement Song," Japan Comment The community commented: "Teresa Teng's accomplishments in Japan's performing arts, even in the hometown of Japan, are top-notch."

Teresa Teng is not only a fusion of Asian music style, but also to absorb the popular elements of Europe and the United States, Teresa singing unique American country music will be the essence of music in Asia, Europe and the United States together to achieve a singing breakthrough.

\section{The Singing Features of Teresa Teng's Songs}

\subsection{The Sound Characteristics of Teresa Teng's Songs}

Teresa Teng singing "sobbing" is one of the most significant artistic features, but also Teresa singing is different from other singer's most significant symbol. Compared with other singers of the same era, Teresa Teng's voice conditions do not dominate, the appearance conditions are more general, according to the usual practice is difficult in the highly competitive pop music in Taiwan and even Southeast Asia occupy a place. Teresa based on their own voice features and singing habits, creative use of the "sobbing" and thus formed its own unique artistic style [3].

"Sobbing" is the process of singing the use of changes in the intensity of breath, singing in the formation of a similar crying voice, to express strong feelings, access to audience understanding and appreciation. Teresa Teng's "Tears of Light" sang with "Weeping Voice", and weepingly told the audience about a beautiful and sad love story. The weeping singing melodiously matched the mood expressed by the song lyrics, and the audiences listen to Teresa Teng "sobbing singing at the same time, more access to the depth of soul resonance.

\subsection{The Language Features of Teresa Teng 's Songs}

Li Yu, a loose-talker in the Qing Dynasty, said: "Students who learn to sing, regardless of skill, only look at those who have no words or mouth. Singing clearly articulate the pronunciation of articulation, whether it is Mandarin songs, Taiwanese songs, Cantonese songs, or Japanese songs, English songs, Teresa Teng can do the word is round, pronunciation standard, and pay attention to the level of inhibition, this is Music in the tone of the language stresses the formation of a phonological beauty [4]. Such as: "Walk Life Road," the song Teresa Teng Japanese and Cantonese versions of the concert, more familiar with the Cantonese version. The music style relaxed and happy, rhythm faster, more short, dense words, Teresa in Cantonese singing is very attention to the granularity of the word and comparison of the word strength, in the change tone "foot" "edge" of the word Increased intensity, accurate pronunciation, clear and rapid, giving a sense of ease.

In the singing of slower songs, such as "the moon represents my heart", "love articles" and so on, through the mouth, pharynx, lips and teeth, tongue, jaw with the Teresa Teng singing will prefix, the 
suffix is very clear, tight and not stiff, loose and relentless, while the support of the breath word, belly, tail conversion is also very coherent. Furthermore, popular songs do not need to be articulated in the resonance cavity like the Western traditional singing style, giving people a sense of distance, and their characters are more natural and close to life. In short, regardless of fast and slow, Teresa Teng in the bite words has done a vivid clear, cadence, showing the rhythm of the language.

\subsection{The Beat Singing Songs of Teresa Teng's Songs}

Teresa Teng pioneered the "Gospel Singing" in mainland China and determined the first pop songs in mainland China

Hand singing style. "Audible sound" state of sound and method is "a certain strength of the air support, half a yawn throat like open, Adam's apple moderate decline, when the breath across the vocal cords, the sound file closed, but cannot be closed too tight too strict, Can be slightly relaxed, gentle pressure, chin to relax, the Adam's apple and vocal cord with the next gas, the size of the file gas pressure and pressure to adapt to the use of vocal mix, biting words close to life, but slightly exaggerated to speak later The resonance of the pharynx resonates, the vocal accompaniment produced by the breath, the vocalization, the bite-stroking, and the acupuncture and acupuncture needs to be accompanied by the sound of the sound. The sound of the sound accompanies the sound of the sound and the image of the sound is graceful, lyrical and emotional [3]. Close to the audience, easy to communicate with the audience.

\subsection{The Emotional Characteristics of Teresa Teng's Songs}

Another major artistic feature of Teresa Teng's songs is their rich, full-bodied feelings. Many artists analyze and study the artistic features of Teresa Teng's song works. Many of her musical works are full of deep, full and sincere feelings, including love and longing for beautiful life, longing for sweet love and attachment to life ideal Praise and praise. Her voice is from the heart and full of emotion, is the soul singer. Her classic songs such as "Dreams in One Curtain", "Town Story" and "The Water Side" are elegant or euphemistic, or enthusiastic. The audience will enjoy deep sympathy in the depths of the soul while listening to the songs. The audience's emotions will be infected and driven by her singing, unconsciously into the mood outlined by her singing, the love and hate of the characters in the song is deeply attracted, this is the concentrated expression of the artistic achievements of the song, is also sought after by many singers goal [4].

\subsection{Singing skills}

Teresa Teng range normally, not very wide, but she is extremely good at mixing true and false singing. Her real singing voice is natural, cordial, close to the style of speaking, the perfect use of its falsetto as a way to modify the sound. This combination of true and false "mixed sound" to the audience a soft, delicate, slightly ethereal artistic effect. In addition, Teresa Teng gentle singing volume, ventilation traces lighter, the overall feeling of more convergence [5]. And, Teresa Teng in the pop scene in Mandarin, the first to use the "singing sound" singing, and singing "sound position" front, uniform atmosphere, better resonance, but also makes her singing soft and not empty. Teresa Teng also used "sobbing" as a means of singing (that is, sobbing and sobbing at the time of singing), which is mainly manifested in the "rising process" at the end of a song or end of song Hiccup "singing), which is also its signature singing characteristics. In addition, Teresa Teng singing good at "tremolo" handling, but also its atmosphere to grasp the better an important manifestation.

\section{The Influence of Teresa Teng Music on Chinese Pop Music}

Teresa Teng's music plays a connecting role in the pop music scene in China. It is a bridge linking the two periods of Chinese pop music before and after. The modern pop singer Teresa Teng's song pioneer and innovative continuation show a wide range of styles.

\subsection{Teresa Teng's position in pop music in China}

Teresa Teng is by far the most successful Chinese music scene, one of the most famous singers, 
she created a song "Teresa Teng era", the Chinese music industry, and especially the development of Chinese pop music plays a decisive role [6].

Teresa Teng is not only a legendary generation of Chinese pop music, but also a symbol of the entire "Chinese pop culture"! Now the goddess in the hearts of the old men who are past Pentecost is always her, only her. These three words are not only a singer's name, but also an era symbol.

From (the moon represents my heart, the town story, sweet honey, last night's stars, a thousand words, I only care about you, where you come again, roadside wild flowers do not adopt), this song is really only heaven, the world can have a few times to hear?

"Before the ancients, but not after" - era of extravagant music, billions of applause remains the same, today seems to be fantastic. Fig.1 shows Teresa Teng portrait.

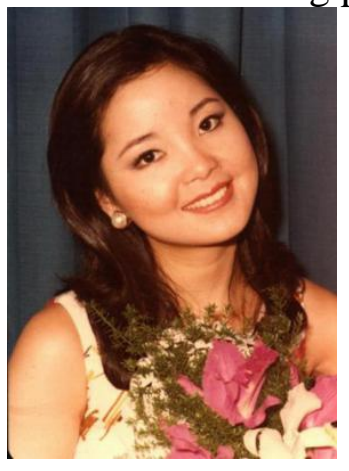

Fig.1 Teresa Teng portrait

\subsection{Create Teresa Teng's musical style}

Teresa's music combines Eastern and Western music elements, the nationality and popularity of fusion, to create their own style characteristics. The general feature of Teresa Teng's singing technique is "singing of the sound of the gas", which achieves the interpretation of the works of different styles through the changes and treatments of the decorative sound, the sound color and the intensity [5]. At the same time, the formation of Teresa Teng's musical style is also inseparable from the features of her singing works. Most of the songs sung by Teresa Teng are steady and slow, with mostly pentas, emphasizing national characteristics and having some common features with her singing style.

\subsection{Continued the history of Chinese pop music}

With the reform and opening up and the beginning of the country, when the mainland was overwhelmed with spiritual needs, Teresa Teng's singing was uninvited and she came to the public's heart. She popularized Chinese pop music to create outstanding works for the first peak period creators and popular singers singing way to show again in front of people, after 30 years of music triggered the "strong horn" the softest part of the public mind. Pop music re-landed in mainland China, and people seem to have felt the popularity of early Chinese pop music in these "Episodes" and gentle graceful, whispered singing styles that Teresa Teng had copied. For the history of Chinese pop music, Teresa Teng continued the re-interpretation of the early popular songs, making the pop music "Great Shanghai Period" without any trace of making and twisting to the period of "Reform and Opening-up," making the two segments separated by thirty in the past year, there was a fusion point and an entrance to popular music without any connection, making people feel that Chinese pop music had stopped, stopped, and only briefly left and shifted. Now it is back again [6].

\subsection{Guided the new era of pop singing in mainland China}

From 1980 to 1985 was the beginning of China's reform and opening up phase, Teresa Teng's songs through the cassette into the tens of thousands of households. At the time of the alternation between the old and new tracks, the song concept and style of enlightenment brought by Teresa Teng led the mainland pop singer to give impetus to the barren and popular pop music of the time, and to imitate the singing and the charm of Teresa Teng became the 1980s early national culture wave [7].

Teresa has promoted the thawing and rebirth of the secular culture in the Mainland and has even 
led directly to the recovery of the popular music industry in the Mainland. Chinese pop music in the effort to imitate I began to explore their own, with its own track. Mr. Kim Siu-kwan concluded: "At this stage, the songs of Hong Kong and Taiwan, and especially the songs of Teresa Teng, have a profound impact on the development of pop music in the Mainland in the future. Her performance has influenced a whole generation of mainland singers [7]. Her orchestra became an imitation of mainland pop music writers Her samples also strongly influenced the writing of a group of mainland composers, and so far, with the introduction of almost all major singers in Hong Kong and Taiwan into the mainland, Teresa still has an irreplaceable status.

\section{Conclusions}

Teresa Teng unique singing style makes Teresa Teng dominate the music scene, loved by the majority of the audience, the audience, her singing art stems from the fertile land of the Chinese nation, with a strong ethnic color to draw the national spirit. Over the past 30 years, her art of singing has become increasingly mature. Her singing style has also been applied by the original natural and unique soft voice, which eventually led to her singing with scientific, nationality and time characteristics. Unique style, the pursuit of natural, gentle, delicate beauty, her singing is not a part of her independence, confidential thinking and meticulous carving. The performance of both the King is the scene is love, is the human thing, singing in her are full of life and affection, fusion of fresh wind and bone beauty, simple emotional beauty and delicate voice, the beauty of form. Teresa has covered an era with singing. She is only a singer, but she has a strong and lasting influence on the development of pop music in China. She is a representative of the historical period and represents the voice of folk in China.

\section{References}

[1] S.T. Li, Teresa Teng - landmark milestone of the 20th century pop music in China, Shenyang: Shenyang Normal University, 2012, vol.14, pp.32-35.

[2] Y.Q. Lu, Teresa Teng's art of singing, Art education, 2010, vol.8, pp.7-10.

[3] M.T. Wang, Teresa Teng Artistic Analysis of Songs, Hua Zhang, 2013, vol.7, pp.21-23.

[4] S.T. Li, Teresa Teng exploration of musical epidemic factors, The sound of the Yellow River, 2011, vol.5, pp.110-112.

[5] C.L. Wang and X.L. Wang, Teresa Teng on the development of Chinese pop music, Economic Research Guide, 2011, vol.10, pp. 246-247.

[6] Y.H. Chen, Teresa Teng Aesthetic Studies in pop Music, Shenzhou, 2011, vol.29, pp.167-170.

[7] H.F. Hong, Teresa Teng Mandarin Pop History and Historical Memory, Singing Art, 2013, vol.3, pp. 20-23. 